Related content

Three-dimensional study of macro- and mesosegregation formation in a rectangular cavity cooled from one vertical side

To cite this article: V F De Felice et al 2012 IOP Conf. Ser.: Mater. Sci. Eng. 33012088

View the article online for updates and enhancements.
- Influence of Discretization of Permeability
$\frac{\text { Term and Mesh Size on the Prediction of }}{\text { Channel Segregations }}$
A Kumar, B Dussoubs, M Založnik et al.
- Analysis of a numerical benchmark for
$\frac{\text { columnar solidification of binary alloys }}{\text { H Combeau, M Bellet, Y Fautrelle et al. }}$
- Numerical study of the impact of inoculant
$\frac{\text { and grain transport on macrosegregation }}{\text { and microstructure formation during }}$
$\frac{\text { solidification of an Al-22\%Cu alloy }}{\text { K O Tveito, M Bedel, M Založnik et al. }}$ 


\title{
Three-dimensional study of macro- and mesosegregation formation in a rectangular cavity cooled from one vertical side
}

\author{
V F De Felice ${ }^{1}, \mathrm{~K}$ O Tveito ${ }^{2}$, M Založnik $^{1}$, H Combeau ${ }^{1}$, M M'Hamdi $^{2,3}$ \\ ${ }^{1}$ Institut Jean Lamour, CNRS - Université de Lorraine, Département SI2M, \\ Ecole des Mines de Nancy, Parc de Saurupt CS14234, F-54042 Nancy cedex, France \\ ${ }^{2}$ Dept. of Materials Technology, NTNU, N-7491 Trondheim, Norway \\ ${ }^{3}$ SINTEF Materials and Chemistry, N-0314 Oslo, Norway
}

E-mail: valerio.de-felice@ijl.nancy-universite.fr

\begin{abstract}
In metal alloys the solidification process leads to heterogeneities in chemical composition, which result in macroscopic (on the scale of the product) and mesoscopic (on the scale of several grains) segregation structures in the casting. Mesosegregations are a severe form of segregation in which solute concentration changes abruptly with respect to the surrounding regions and can induce structural heterogeneities in the casting. In this paper we move on from $[1,2]$, in which channel mesosegregations and their numerical prediction were studied on a two-dimensional configuration - a small $(10 \times 6 \mathrm{~cm})$ ingot of a $\mathrm{Sn}-10 \mathrm{wt} \% \mathrm{~Pb}$ binary alloy. We extend the study to a slender three-dimensional cavity $(10 \times 6 \times 1 \mathrm{~cm})$ and we investigate three-dimensional effects in the formation of channel mesosegregations in a columnar mushy zone. We investigate the effects of the three-dimensionality on the structure of channels in the mushy zone and of channel mesosegregations. We present the influence of the permeability of the mushy zone on the channel structures. We show that channels can form either as lamellar or as tubular structures: at higher permeability secondary instabilities across the cavity thickness promote a transition from lamellar to tubular channels.
\end{abstract}

\section{Introduction}

The prediction and the control of chemical, structural and mechanical heterogeneities represent a fundamental target in metal processing. In solidification processes heterogeneities of chemical composition inevitably form. They appear as macroscopic (on the scale of the product) and mesoscopic (on the scale of several grains) segregation structures. Macro- and mesosegregation in the casting severely alter the homogeneity and the quality of the final product. Mesosegregation represents a severe form of segregation in which solute concentration varies abruptly in a certain part of the casting. These strong chemical variations can induce structural heterogeneities of the casting that impair its mechanical properties. Channel mesosegregations appear as long narrow trails of strong segregation aligned in some preferred direction. The precise mechanism of their formation is yet unexplained. In this frame, both experimental studies and numerical simulations are needed to better understand the segregation mechanisms and provide a tool to control and to minimize their effect.

The evolution of segregation depends on the entire temporal history of the solidification process, which presents a strong coupling between the momentum, mass and thermal transport. Perturbations in the mushy zone due to the liquid flow and due to the transport of solute seem to be the principal phenomena leading to instabilities that develop into channel mesosegregation structures. Accurate simulation and analysis of the fluid flow, thermal gradients and solute transport, are crucial in the 
investigation of channel genesis. In this work, we move from the results of $[1,2]$, where mesosegregation for a two-dimensional rectangular cavity $(10 \times 6 \mathrm{~cm})$ filled by a liquid $\mathrm{Sn}-10 \mathrm{wt} \% \mathrm{~Pb}$ binary alloy was studied, and we extend the study to a three-dimensional cavity $(10 \times 6 \times 1 \mathrm{~cm})$. Some recent simulation results for this three-dimensional configuration, presented in [3], showed that mesosegregations form as lamellae, basically retaining a two-dimensional structure. Experimentally, tubular channel mesosegregations were observed on different configurations, notably in paper [4], where experimental investigations by X-ray tomography and 3D simulations were performed for a small Sn-Bi alloy casting. In the present work we suggest the existence of a transition in the mesosegregation morphology. We indicate that the influence of secondary three-dimensional flows could break up the two-dimensional lamellar structure and develop tubular channel mesosegregation structures. We study the lamellar-to-tubular transition as a function of the DAS (dendrite arm spacing) and show that at higher DAS (and thus higher permeability of the mushy zone) instabilities in the transversal direction, inducing tubular structures, are more likely to develop.

We present the problem configuration in section 2 . The governing equations as well as the numerical resolution procedure are detailed in sections 3 and 4; and the three-dimensional effects in the formation of channel mesosegregations in a columnar mushy zone are presented in section 5 .

\section{Problem setup}

The solidification of a binary $\mathrm{Sn}-10 \mathrm{wt} \% \mathrm{~Pb}$ alloy in a three-dimensional cavity $(10 \times 6 \times 1 \mathrm{~cm})$ is simulated. The cavity is symmetrically cooled from its two vertical small faces, by an external flow at the temperature $T_{\infty}$ and heat transfer coefficient $\alpha$. The cooling is described by identical Fourier boundary conditions on the two external vertical walls. The top, bottom, front and back walls are thermally insulated. No-slip boundary conditions are imposed at the walls. We assumed symmetry of solution with respect to Plane S (see Figure 1) and we solved the problem on a half of the entire ingot volume. The ingot cavity is initially filled with the liquid alloy at rest; the alloy is at its nominal composition and at the liquidus temperature $T_{l i q}=219.14^{\circ} \mathrm{C}$.

Table 1. Properties of Sn-10wt.\% Pb, boundary and initial conditions

\begin{tabular}{llr}
\hline Symbol & Units & Value \\
\hline$c_{p}$ & $\mathrm{~J}\left(\mathrm{~kg}^{\circ} \mathrm{C}\right)^{-1}$ & 260 \\
$k$ & $\mathrm{~W}\left(\mathrm{~m}^{\circ} \mathrm{C}\right)^{-1}$ & 55.0 \\
$\rho_{0}$ & $\mathrm{~kg} \mathrm{~m}^{-3}$ & 7000 \\
$L$ & $\mathrm{~J} \mathrm{~kg}^{-1}$ & $6.1 \times 10^{4}$ \\
$\mu_{l}$ & $\mathrm{~Pa} \mathrm{~s}$ & $1.0 \times 10^{-3}$ \\
$\beta_{T}$ & ${ }^{\circ} \mathrm{C}^{-1}$ & $6.0 \times 10^{-5}$ \\
$\beta_{C}$ & $(\mathrm{wt} \%)^{-1}$ & $-5.0 \times 10^{-3}$ \\
$T_{m}$ & ${ }^{\circ} \mathrm{C}$ & 232.0 \\
$C_{e}$ & $\mathrm{wt} \%$ & 38.1 \\
$k_{p}$ & - & 0.0656 \\
$m_{l}$ & ${ }^{\circ} \mathrm{C}(\mathrm{wt} \%)^{-1}$ & -1.286 \\
$C_{0}$ & $\mathrm{wt}$ & 10.0 \\
$T_{0}=T_{l i q}$ & ${ }^{\circ} \mathrm{C}$ & 219.14 \\
$\alpha$ & $\mathrm{Wm}^{-2 \circ} \mathrm{C}^{-1}$ & 400 \\
$T_{\infty}$ & ${ }^{\circ} \mathrm{C}$ & 25 \\
\hline
\end{tabular}
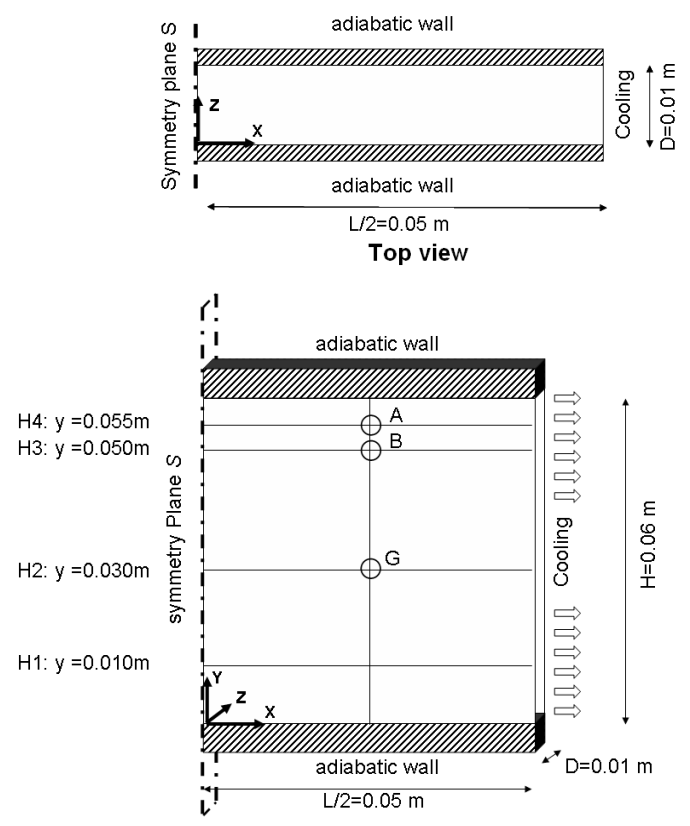

Figure 1. Problem geometry setup. 
Once the solidification process is started, the solid phase grows in a columnar mushy zone that forms on the right hand side of the model geometry, moving from the cooled side toward the symmetry plane. A liquid motion is induced, driven by both thermal and solutal buoyancy.

\section{Mathematical modelling}

The solidification process in multi-component systems involves the presence of both liquid and solid phases as well as a mushy zone. In literature the governing equations are often derived moving from the basic two-phase flow formulation, where the equations for each phase are additively combined taking into account the interactions at the liquid-solid interface (we refer the reader to references $[5,6]$ for further details). In this frame the balance equations (Eqs. (1-3)) for the transport of mass, heat, solute and momentum are presented in order to provide a necessary basis for the subject presented in this work. The microsegregation model (lever rule) corresponds to the assumption of a full thermodynamic equilibrium of the solid and liquid phases (Eq. (6)). The density of the liquid phase in the buoyancy term follows a linear variation with the temperature and the solute concentration (Eq. (4)). The permeability of the porous mushy zone depends on the liquid fraction and the dendrite arm spacing DAS, and follows the Kozeny-Carman law (Eq. (5)). The model is identical to the one defined in the benchmark [1] and more details about the model assumptions are given in [2].

Table 2. Model equations.

\begin{tabular}{l} 
Mass and momentum: $\quad \nabla \cdot \vec{v}=0 \quad \rho_{0} \frac{\partial \vec{v}}{\partial t}+\rho_{0} \nabla \cdot\left(\vec{v} \frac{\vec{v}}{g_{l}}\right)=-g_{l} \nabla p+\mu_{l} \nabla^{2} \vec{v}+g_{l} \tilde{\rho} \vec{g}-\frac{g_{l} \mu_{l}}{K} \vec{v}$ \\
Heat: $\rho_{0} \frac{\partial h_{m}}{\partial t}+\rho_{0} \nabla \cdot\left(\vec{v} h_{l}\right)=\nabla \cdot(k \nabla T)$ \\
Density: $\quad \tilde{\rho}=\rho_{0}\left[1-\beta_{T}\left(T-T_{0}\right)-\beta_{C}\left(C_{l}-C_{0}\right)\right]$ \\
Lever rule: $\langle C\rangle=g_{s} C_{s}+g_{l} C_{l}=C_{l}\left[g_{l}+k_{p}\left(1-g_{l}\right)\right] ; \quad T_{l i q}=T_{m}+m_{l} C_{l} ; \quad h_{m}=g_{s} h_{s}+g_{l} h_{l}=c_{p} T+L$ \\
\hline
\end{tabular}

\section{Solution procedure}

We used two different finite-volume codes to perform the computations. The first code (NTNU/SINTEF) was written at NTNU (Trondheim, Norway), in Fortran 90 and parallelised using MPI. The second code (CastFOAM) was written at IJL (Nancy, France) and is based on the OpenFOAM ${ }^{\circledR}$ platform written in C++ [9]. In the NTNU/SINTEF code, the two-phase solidification model is based on the operator splitting scheme by Založnik et al. [7] for coupling macroscopic transport and grain growth, but in the present work the model is simplified under the assumption of local thermodynamic equilibrium and stationary solid [8]. The system of equations is discretized with the finite-volume method and the SIMPLE [10] method for staggered grids is used for pressurevelocity coupling. The convective terms are discretized with the MUSCL [10] scheme, using the van Leer flux limiter [10] and a first order fully implicit Euler scheme [10] is used for the time derivative. At a given time step, the following four steps are performed successively for the NTNU/SINTEF code until the convergence test in step 5 is satisfied:

1) Resolution of the momentum and global mass balance equations:

The SIMPLE [10] algorithm is used to treat the pressure-velocity coupling; the last known values of $T$ and $C_{1}$ are used in the calculation of the buoyancy term.

2) Resolution of the solute mass balance equation during the transport stage:

The conservation equation of $C_{l}^{t r}$ is solved according to the splitting scheme [7]: 


$$
\begin{aligned}
& \frac{\partial\left(g_{l}^{t r} C_{l}^{t r * * *}\right)}{\partial t}+\nabla \cdot\left(\vec{v} C_{l}^{t r, * *}\right)=0, \quad C_{m}^{* *}=g_{l}^{t r} C_{l}^{t r, * *}+g_{s}^{t r} C_{s}^{t r} \\
& \left(g_{l}, g_{s}, C_{s}\right)^{t r}=\left(g_{l}, g_{s}, C_{s}\right)^{o l d} \quad \text { (stationary solid) }
\end{aligned}
$$

3) Resolution of the energy equation:

The energy equation is solved for the average enthalpy, $h_{m}$. A first order Taylor expansion is used for the temperature to obtain an implicit evaluation of the convection and conduction terms:

$$
\begin{aligned}
& \frac{\partial h_{m}^{* *}}{\partial t}+\nabla \cdot\left(\rho_{0} c_{P} \vec{v} \tilde{T}\right)=\nabla \cdot(k \nabla \tilde{T}) \\
& \tilde{T}=T^{*}+\frac{d T}{d h}\left(h_{m}^{* *}-h_{m}^{*}\right), \quad \frac{d T}{d h}=\max \left(\frac{1}{10 c_{p}}, \frac{T^{*}-T^{\text {old }}}{h_{m}^{*}-h_{m}^{\text {old }}}\right)
\end{aligned}
$$

4) Local equilibrium:

Under the assumption of local equilibrium the liquid and solid solute concentration, liquid fraction and temperature are calculated from the new estimation of $h_{m}$ and $C_{m}=g_{s}^{t r} C_{s}^{t r}+\mathrm{g}_{l}^{t r} C_{l}^{t r}$. Equilibrium is defined by Eqs. (6). To solve this system, they are combined into a quadratic equation, which is solved analytically for the liquid fraction $g_{1}$ :

$$
\begin{aligned}
& a\left(g_{l}^{*}\right)^{2}+b g_{l}^{*}+c=0 \\
& a=\left(k_{p}-1\right) L ; \quad b=\left(k_{p}-1\right)\left(c_{p} T_{m}-\langle h\rangle\right)-k_{p} L ; \quad c=k_{p}\left(\langle h\rangle-c_{p} T_{m}\right)-\left(c_{p} m C\right)
\end{aligned}
$$

5) Convergence test:

Steps 1-4 are repeated until the following convergence criterion is satisfied:

$$
\max \left|\frac{|\vec{v}|^{* *}-|\vec{v}|^{*}}{f_{\text {velocity }}^{\text {norm }}}\right|<\varepsilon, \max \left|\frac{p^{* *}-p^{*}}{f_{\text {pressure }}^{\text {norm }}}\right|<\varepsilon, \max \left|\frac{C_{l}^{t r, * *}-C_{l}^{t r, *}}{f_{\text {solute }}^{\text {norm }}}\right|<\varepsilon, \max \left|\frac{h_{m}^{* *}-h_{m}^{*}}{f_{\text {enthalpy }}^{\text {norm }}}\right|<\varepsilon
$$

where $f^{\text {norm }}$ is a normalization factor for each equation. When the criterion is satisfied the time is advanced by $\Delta t$; the symbol $*$ denotes the value of the last iteration and $* *$ the value of the new iteration of the equation system.CastFOAM employs a different scheme for coupling transport and phase change. In each time step, the momentum and continuity equations are solved first in a step of the PISO [10] pressure-velocity coupling scheme. Then the solute conservation equation is solved in terms of the liquid concentration $C_{1}$. After that thermodynamic equilibrium (lever rule) is recovered by solving Eqs. (6). Then the heat transport equation in solved in a temperature formulation and after that the lever rule (Eqs. (6)) is applied again. This is iterated until convergence is reached and the time is advanced by $\Delta t$. The discretization is formulated on a collocated pressure-velocity arrangement, the convective terms are discretized with the QUICK [10] scheme and implicit Euler [10] time stepping is used.

\section{Results and discussion}

The results of simulations of solidification in the three-dimensional ingot, obtained with the NTNU/SINTEF code, are presented in this section. First, simulation results, for a test case, of the NTNU/SINTEF code are compared and validated with CastFOAM. Results in terms of macro- and meso-segregation at the end of solidification are discussed in the two cases. Then a parametric study in terms of dendrite arm spacing is performed in order to show the effects of permeability (Eq. (5)) in the mushy zone on the segregation behaviour. For the simulations a constant time step $\Delta t=0.005 \mathrm{~s}$, much smaller than the solidification, convection and diffusion characteristic times, was chosen. For the space-discretization a regular cubic mesh was chosen for the cavity: $128 \times 128 \times 32$ volumes. The simulations end at $t=600 \mathrm{~s}$, after that the entire ingot is completely solidified. According to [1] and [11], the choice of these parameters provide a good spatial and time resolution, necessary for the 
description of the mesosegregation zone, where the shape, the length and the position of channels are very sensitive to mesh size [11].

\subsection{Comparison of NTNU/SINTEF code and CastFOAM results: concentration field at the end of} solidification and characterization of the mesosegregation zones.

We investigate the results at the end of solidification obtained by the two codes for Case 1 (Table 3 ). We first present the segregation map at the end of solidification ( $t=600 \mathrm{~s})$ in the vertical XY symmetry plane $(z=0.005 \mathrm{~m})$ obtained by the two codes.

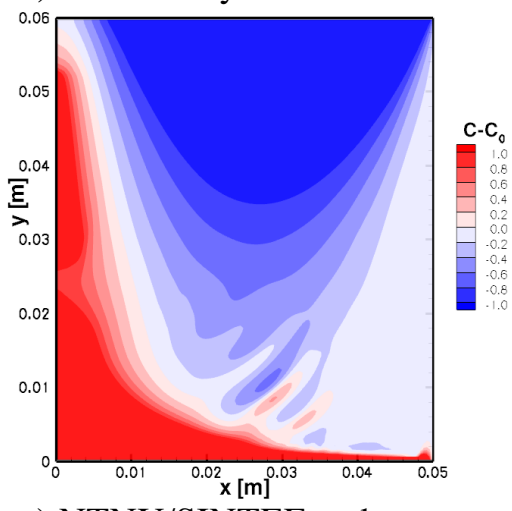

a) NTNU/SINTEF code

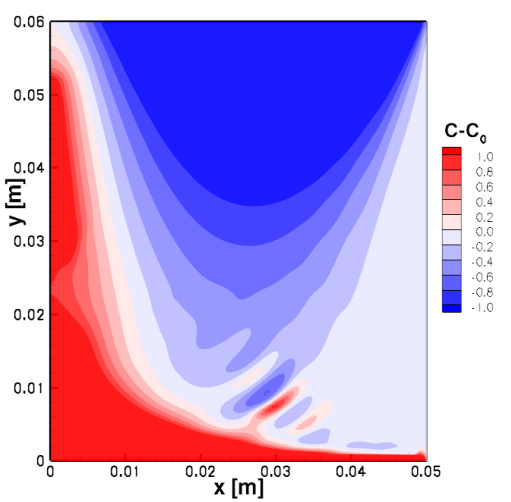

b) CastFOAM

Figure 2. Segregation $\left(C-C_{0}\right.$ in $\left.\mathrm{wt} \% \mathrm{Sn}\right)$ at $t=600 \mathrm{~s}$ in the vertical mid-section obtained by the NTNU/SINTEF code and by CastFOAM.

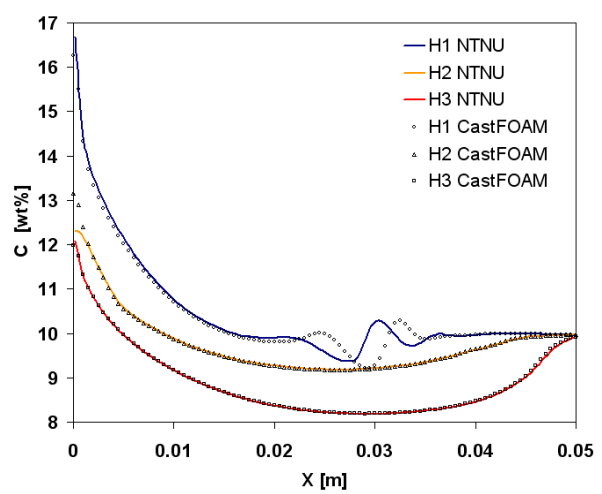

Table 3. Dendrite arm spacing (DAS) used in the four simulations.

\begin{tabular}{lr}
\hline Case & DAS \\
\hline 1 & $65 \mu \mathrm{m}$ \\
2 & $90 \mu \mathrm{m}$ \\
3 & $120 \mu \mathrm{m}$ \\
4 & $150 \mu \mathrm{m}$ \\
\hline
\end{tabular}

Figure 3. Average solute concentration $(C$ in $\mathrm{wt} \% \mathrm{~Pb})$ profiles along the $\mathrm{x}$ direction in sections $\mathrm{H} 1, \mathrm{H} 2$ and $\mathrm{H} 3$ obtained by NTNU/SINTEF code (continuous lines) and CastFOAM (symbols) in the symmetry plane for Case 1 .

Figure 2 shows a large negative segregation zone in the central and the upper regions of the domain, while in the bottom and the left part of the domain a large positive segregation region is observed. This is a classical result for columnar solidification: negatively segregated zones form where the liquid flow is oriented opposite to the temperature gradient and positively segregated zones, where it is oriented in the same direction. The cooling here is from the right and the natural convection creates a clockwise flow circulation. Additionally, the liquid that solidified last (on the left) has been progressively enriched in $\mathrm{Pb}$ content, which generates a positively segregated zone along the left vertical boundary. In bottom of the domain we observe a mesosegregated region in which narrow channels appear (aligned at about $45^{\circ}$ ). Inside them, the solute concentration is significantly higher than in the surrounding regions. The comparison between the results suggests that the global macrosegregation patterns have similar behaviour. In the meso-segregation region we observe, in both cases, the formation of the same number of channel mesosegregations with approximately the same orientation, length and location. The mesosegregation intensity prediction, however, is higher in CastFOAM, 
while the NTNU/SINTEF code predicts longer channels. Concentration profiles along sections H1, H2 and $\mathrm{H} 3$ (see Figure 1) are presented in Figure 3 and show in a more quantitative way the good agreement in macrosegregation prediction and the presence of the same number of mesosegregations, although a shift of their $x$ position is observed. The observed small differences in mesosegregation patterns provided by the two codes are similar to the differences already observed between the software tested in the frame of the numerical benchmark on macro- and mesosegregation prediction [1]. As we will show, the structures in Case 1 are practically two-dimensional and only little variation is observed along the thickness ( $z$ direction). Comparison of results in three dimensions provided by the two codes for the remaining three cases also provided good agreement: very close segregation structures and lamellar-to-tubular channel transitions were obtained. Results obtained by the Leoben group [3] (only for Case 1) also agreed and they showed a lamellar structure of mesosegregates.

\subsection{Influence of permeability on the 3D structure of the channel mesosegregates}

The dendrite arm spacing (DAS) represents a crucial parameter in solidification calculations because it strongly affects the permeability (Eq. (5)) in the mushy zone. In this section a parametric study on DAS is performed and shows that as this parameter increases, the increase of permeability strongly affects the segregation evolution, leading to modifications in the macro- and mesosegregation in the ingot. Results for four values of DAS (Table 3) are presented in terms of average solute concentration at the end of solidification. In Figure 4, in order to show the three dimensional structure of the channel mesosegregates, an isosurface of the average concentration is presented and confirms that in Case 1, corresponding to the smallest DAS, the segregates are organised in form of lamellae that are close to being two-dimensional. Only a small three-dimensional effect is noticed close to the lateral walls. In Figure 4(a) we can see that for small DAS the channel mesosegregations are lamellar. In Figure 4(b) the results for the transition Case 2, corresponding to higher permeability, are presented: the two dimensional structure of the lamellae is broken and a central tubular structure appears. In Figures 4(c), and 4(d), corresponding to even higher values of DAS, the average concentration isosurfaces show the presence of a complex mesosegregate with secondary tubular structures, which branch from the central tubular channel already observed in Figure 4(b).

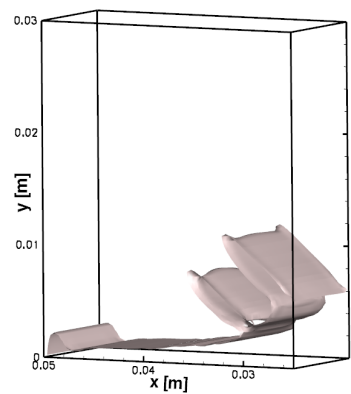

(a) Case 1 $(\mathrm{DAS}=65 \mu \mathrm{m})$ : lamellar

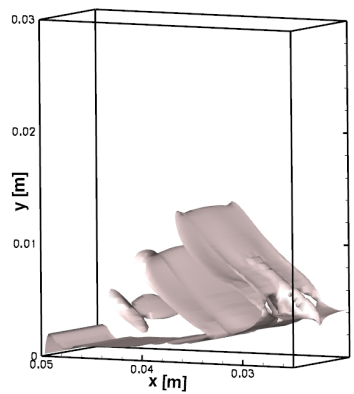

(b) Case 2 (DAS = 90 $\mu \mathrm{m})$ : lamellar-tubular

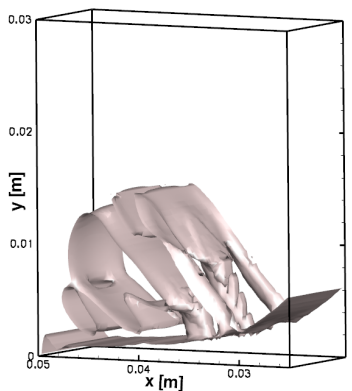

(c) Case 3

(DAS $=120 \mu \mathrm{m})$ : lamellar-tubular

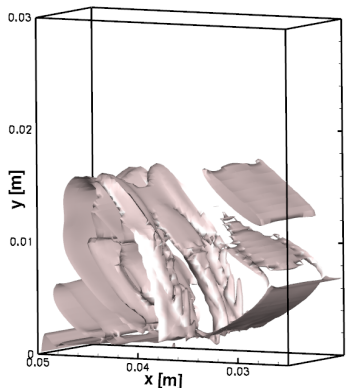

(d) Case 4 (DAS $=150 \mu \mathrm{m})$ : branched tubular

Figure 4. Transition of the channel mesosegregation structure with increasing DAS. The segregation isosurface $\left(C-C_{0}=0.1\right)$ at the end of solidification is shown.

The results of the simulations suggest a lamellar-to-tubular transition in the range of DAS [65:90 $\mu \mathrm{m}]$; from quasi two-dimensional lamellar channel mesosegregates to a fully three-dimensional structure, which increases in complexity as the DAS increases.

\subsection{Lamellar to tubular transition in Case $2(D A S=90 \mu \mathrm{m})$}

In this section we analyse the destabilisation of the lamellar channel structure and the formation of the inner segregation channel for Case 2 (Figure 4(b)). In Figure 5 the formation of the channels is 
presented by a sequence of temporal snapshots. Three isosurfaces for the liquid fraction are shown: $g_{1}=0.90$ (light blue), $g_{1}=0.95$ (pink) and $g_{1}=0.98$ (yellow).

In Figures 5(a) and (b) we observe a first instability in the solidification front that occurs in the XY plane where the first lamella is forming. Its shape is practically two-dimensional, slight threedimensional effects are only seen at the walls; they occur due to the delay of the front close to the boundaries. The mechanism seems to follow the same behaviour as described for the $2 \mathrm{D}$ case in $[1,2]$ inducing a lamellar-type effect. At $t=34 \mathrm{~s}$, the $g_{1}=0.98$ isosurface already presents transversal perturbations. During the time evolution (Figures 5(b)-(f)) these perturbations are damped however, and the surface becomes flat again. A delay of solidification close to the lateral boundaries is due to the recirculating flow that is more important in the inner part of the domain and promotes the cooling effect and the solidification.

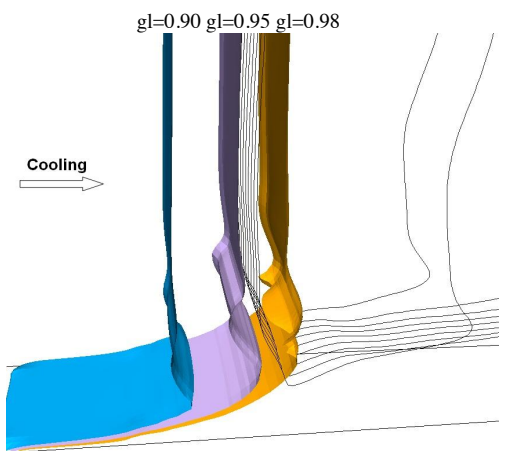

(a) $t=34 s$

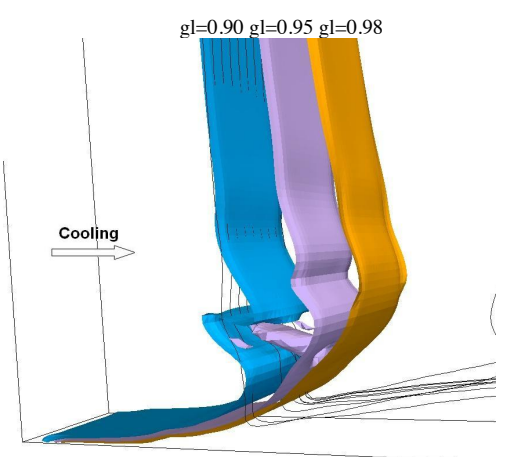

(d) $\mathrm{t}=51 \mathrm{~s}$

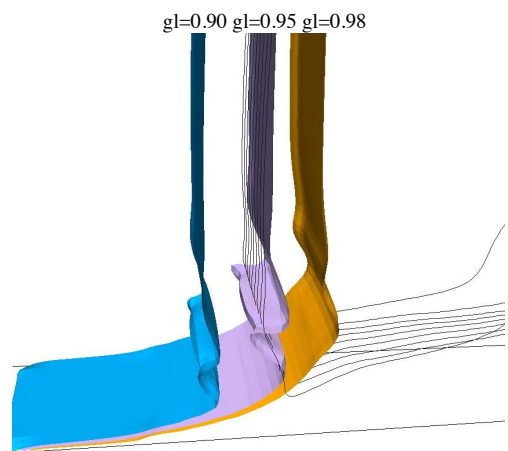

(b) $t=39 \mathrm{~s}$

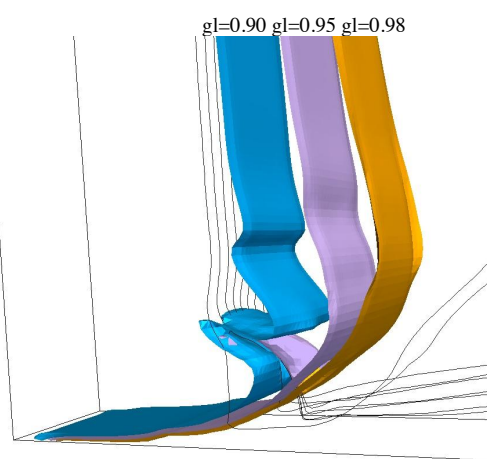

(e) $t=58 \mathrm{~s}$

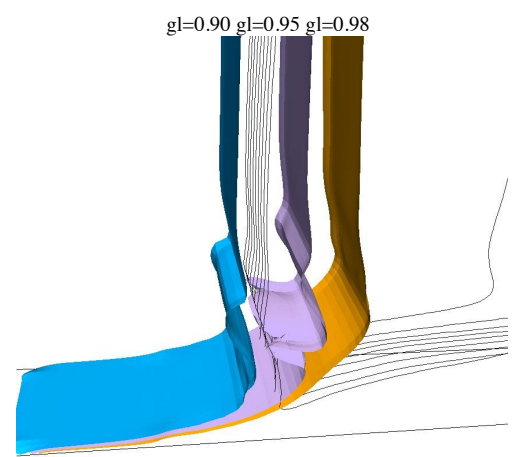

(c) $t=45 \mathrm{~s}$

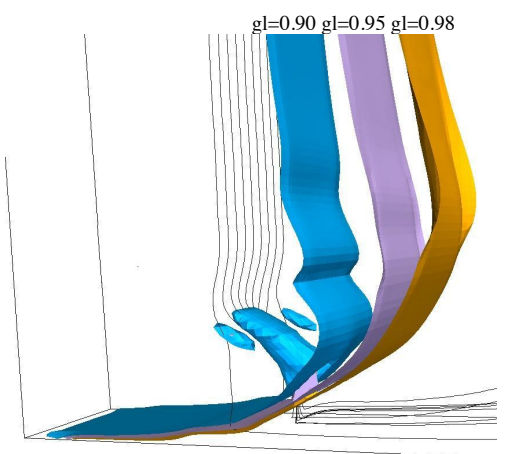

(f) $\mathrm{t}=70 \mathrm{~s}$

Figure 5. Transition form lamellar to tubular channels for Case 2. Isosurfaces of liquid fraction and streamlines of liquid velocity are shown. $g_{1}=0.90$ (light blue), $g_{1}=0.95$ (pink) and $g_{1}=0.98$ (yellow).

In Figure 5(c) a second instability in the isosurface $g_{1}=0.95$ occurs in the $z$ direction. A central lamella and two lateral channels develop. This is an instability that leads to a lamellar-to-tubular transition, which progressively develops the tubular structures. In Figure 5(d), at $g_{1}=0.95$, it developed into a clear central tubular channel. At the same moment a lamellar shape is observed at $g_{1}=0.90$. We can see that streamlines now concentrate in the central part of the domain due to the higher permeability of the tubular channel, which promotes the passage of the fluid flow. The $g_{1}=0.98$ isosurface is still flat.

Between $t=51 \mathrm{~s}$ and $58 \mathrm{~s}$ the $g_{1}=0.98$ isosurface is very quickly destabilized. In Figures $5(\mathrm{e})$ and (f) all three isosurfaces show the presence of a central tubular channel, which becomes the preferred passage for the flow - almost all the streamlines are concentrated there. As a conclusion, for the Case 2 , the analysis of the mesosegregate evolution shows a regular lamellar to tubular transition - a result of a three-dimensional instability of the solidification front. 


\section{Conclusion}

This work presents macro- and mesosegregation in a three-dimensional cavity filled by a $\mathrm{Sn}-10 \mathrm{wt} \% \mathrm{~Pb}$ binary alloy and shows the sensibility of results with respect to the dendrite arm spacing. The structure of the mesosegregation pattern is presented in three dimensions, and major differences for four different DAS are shown at the end of solidification. The study of the evolution of the system in the DAS $=90 \mu \mathrm{m}$ case (Case 2) for the first 70 s suggests that after a first instability of the solidification front, which generates lamellar, quasi two-dimensional channel mesosegregation structures, a second instability of the front in the transversal direction is responsible for the development of fully threedimensional tubular structures.

\section{Acknowledgement}

The Norwegian partners acknowledge support by Hydro, Alcoa Norway, Aleris, the Research Council of Norway and NOTUR High Performance Computing program.

\section{References}

[1] Combeau H, Bellet M, Fautrelle Y, Gobin D, Arquis E, Budenkova O, Dussourbs B, Duterrail Y, Kumar A, Mosbah S, Quatravaux T, Rady M, Gandin C.-A, Goyeau B, Založnik M 2011 Frontiers in Solidification Science, TMS Annual Meeting 2012 (San Diego (CA), USA) (TMS, Warrendale (PA), USA) p 755

[2] Bellet M, Combeau H, Fautrelle Y, Gobin D, Arquis E, Budenkova O, Dussourbs B, Duterrail Y, Kumar A, Mosbah S, Rady M, Gandin C.-A, Goyeau B, Založnik M 2009 Int. J. Therm. Sci. 482013

[3] Li J, Wu M, Hao J, Ludwig A, 2012 IOP Conf. Series: Mater. Sci. Eng. 27 (2011) 012055.

[4] Sawada T, Oikawa K, Anzai K, 2009 International Conference on Simulation and Modelling of Metallurgical Processes in Steelmaking (Steelsim) (Leoben, Austria)

[5] Ni J, Beckermann C, 1991 Metall. Trans. B 22349

[6] Bennon W D, Incropera F P, 1987 Int. J. Heat Mass Transfer 302161

[7] Založnik M, Combeau H 2010 Comput. Mater. Sci. 481

[8] Založnik M, Kumar A, Combeau H 2010 Comput. Mater. Sci. 4811

[9] OpenFOAM ${ }^{\circledR}$, version 1.7.1, computer program, OpenCFD Inc.

[10] Prosperetti A, Tryggvason G, Computational Methods for Multiphase flow, 2007, Cambridge University Press

[11] Kumar A, Dussoubs B, Založnik M, Combeau H 2009 J. Phys. D: Appl. Phys. 42105503 